Characterization of the DT Ice Layer in a Fusion Capsule Using a

Two-Dimensional X-Ray Shearing Interferometer

K. L. Baker

June 6, 2011

SPIE Optics and Photonics

San Diego, CA, United States

August 1, 2010 through August 5, 2010 
This document was prepared as an account of work sponsored by an agency of the United States government. Neither the United States government nor Lawrence Livermore National Security, LLC, nor any of their employees makes any warranty, expressed or implied, or assumes any legal liability or responsibility for the accuracy, completeness, or usefulness of any information, apparatus, product, or process disclosed, or represents that its use would not infringe privately owned rights. Reference herein to any specific commercial product, process, or service by trade name, trademark, manufacturer, or otherwise does not necessarily constitute or imply its endorsement, recommendation, or favoring by the United States government or Lawrence Livermore National Security, LLC. The views and opinions of authors expressed herein do not necessarily state or reflect those of the United States government or Lawrence Livermore National Security, LLC, and shall not be used for advertising or product endorsement purposes. 


\title{
Characterization of the DT Ice Layer in a Fusion Capsule Using a Two-Dimensional X-Ray Shearing Interferometer
}

\author{
K.L. Baker \\ Lawrence Livermore National Laboratory, 7000 East Ave., Livermore, CA, USA 94550
}

\begin{abstract}
This article presents the design and simulated performance of a two-dimensional x-ray shearing interferometer wavefront sensor. In particular, this phase sensitive x-ray wavefront sensor is evaluated for its ability to perform metrology on the DT ice layer in an inertial confinement fusion capsule. The interferometer uses crossed phase gratings in a single plane and is capable of operation over a wide range of x-ray energies by varying the grating material and thickness. The wave-front sensor is insensitive to vibrations and, unlike X-RayTalbot interferometers, recovers the full two-dimensional phase profile of the x-ray beam rather than the gradient in only one dimension.
\end{abstract}

Keywords: Metrology, X-rays, shearing interferometer, wave-front sensing

\section{INTRODUCTION}

Great strides have been made in the pursuit of fusion energy as a relatively clean and essentially unlimited energy source over the past half century. One of the two approaches to achieving fusion energy, inertial confinement fusion(ICF), uses a large number of lasers to either directly compress a capsule containing Deuterium and Tritium(DT) or to indirectly convert the laser photons into x-rays on the wall of a hohlraum and use these x-rays to compress a capsule filled with DT placed within the hohlraum. The latter indirect drive ICF is currently being pursued by the newly commissioned National Ignition Facility, NIF, which is attempting to achieve scientific breakeven beginning in the summer of 2010. In order for the NIF to achieve ignition the illumination symmetry must be carefully controlled. This illumination symmetry includes the power balance and temporal intensity profile between each of the beam lines as well as their position on the hohlraum walls which must be kept to within $~ 50$ microns RMS of their assigned position. In addition to the power balance and position of each of the laser beams, the fusion capsule itself must meet rigorous requirements in order for a particular ignition shot to be successful. A generic fusion capsule is spherical in shape, $\sim 2 \mathrm{~mm}$ in diameter, and consists of an outer ablator shell of $\mathrm{CH}$ and an inner layer of DT ice. The DT ice layer in the fusion capsule contains ice grain boundaries which range between 1 to $10 \mu \mathrm{m}$ in depth. ${ }^{1}$ Based on experimental data it is believed that the maximum grain boundary depth that can be tolerated on a fusion shot is $\sim 5 \mu \mathrm{m}$ without unacceptably impacting the yield. It is therefore desired to reject all targets which have ice grain boundary depths that exceed this value. A method is therefore needed to measure the phase profile of a given capsule to determine if any of the ice grain boundaries present in the DT layer exceed this depth. There is also a window of time between when a fusion capsule is filled with DT and when the shot is taken which is bracketed by multiples of the DT layering time, $30 \mathrm{~min} .{ }^{2}$ constant and the time over which too much ${ }^{3} \mathrm{He}$ is accumulated. Measurements must be taken in situ after the tritium beta decay has had time to produce a roughly uniform layer of DT ice but can't take an excessively long time such that too much ${ }^{3} \mathrm{He}$ has accumulated which can also prevent a successful ignition shot from occurring.

The subject of this article deals with the metrology of the fusion capsule itself. In particular on a two-dimensional xray wavefront sensor that is capable of measuring the wavefront gradient of these ice grain boundaries, reconstructing the two-dimensional phase and based on the amplitude of that reconstructed phase making a decision as to whether the fusion capsule meets the requirements necessary for the fusion capsule to perform as required for the ignition shot. The design and simulation of the expected performance of a two-dimensional x-ray shearing interferometer wavefront sensor is presented below. The interferometer uses crossed phase gratings in a single plane and is capable of operation over a wide range of $\mathrm{x}$-ray energies by varying the grating material and thickness. ${ }^{3,4}$ 


\section{SETTING UP THE SIMULATIONS}

\subsection{DT Ice Grain Boundaries}

The fusion capsules are spherical in shape and consist of an outer ablator shell with an inner layer of DT fuel. The fusion capsules have an overall diameter of approximately $2 \mathrm{~mm}$. In the case of an outer $\mathrm{CH}$ ablator, the thickness of the ablator shell is approximately $150 \mu \mathrm{m}$ and the DT layer on the inside of the capsule is approximately 65 microns thick. At x-ray wavelengths the index of refraction is expressed as $n=(1-\delta)+i \beta$, where $1-\delta$ gives rise to a phase shift as the $\mathrm{x}$-rays pass through the sample and the $\beta$ term results in absorption. The length for a $\pi$ phase shift, $x_{\pi}$, is expressed as $x_{\pi}=\lambda /(2 \delta)$ and the absorption length, $x_{\mu}$, is written as $x_{\mu}=\lambda /(4 \pi \beta)$. At an $x$-ray energy of $10 \mathrm{keV}$, the $\mathrm{x}$ rays have a wavelength of $\lambda=1.24 \times 10^{-10} \mathrm{~m}$. The phase shift due to the $\mathrm{CH}$ ablator, $1.1 \mathrm{~g} / \mathrm{cm}^{3}$, at this wavelength is $\mathrm{x}_{\pi}=\lambda /(2 \delta), \delta=2.5 \times 10^{-6}$, or a $\pi$ phase shift over a distance of $25 \mu \mathrm{m}$ and the phase shift due to the DT, $0.101 \mathrm{~g} / \mathrm{cm}^{3}$, at this wavelength is $\mathrm{x}_{\pi}=\lambda /(2 \delta), \delta=$ 4.x $10^{-7}$, or a $\pi$ phase shift over a distance of $150 \mu \mathrm{m}$. The $\mathrm{CH}$ represents a line-integrated depth of $\sim 300 \mu \mathrm{m}$ or 38 radians and the DT represents a line-integrated depth of $130 \mu \mathrm{m}$ or 2.6 radians. The contribution from both sides of the $\mathrm{CH}$ ablator and from both DT ice layers yields a combined phase shift of $\sim 40.6$ radians. The DT ice layer in the fusion capsule forms grain boundaries which range between 1 to $10 \mu \mathrm{m}$ in depth. ${ }^{1}$ Based on experimental data it is believed that the maximum grain boundary depth that can be tolerated on a fusion shot is $\sim 5 \mu \mathrm{m}$ without unacceptably impacting the yield. ${ }^{1}$ It is therefore desired to reject all targets which have grain boundary depths that exceed this value. A method is therefore needed to measure the phase profile of a given capsule to determine if any of the grain boundaries present in the DT layer exceed this depth. A $5 \mu \mathrm{m}$ depth at the grain boundary in the DT ice layer would then make a difference in the phase of $5 \mu \mathrm{m}$ out of the line-integrated DT depth of $130 \mu \mathrm{m}$. This is on top of an $\sim 0.5 \mu \mathrm{m}$ RMS surface roughness for the DT ice layer. ${ }^{1}$ That represents only a 3.8\% difference in the path length or in the phase shift, $0.098 \mathrm{rad}$, for the crevice and $0.4 \%$ or 0.0098 rad RMS due to the surface roughness. The wavefront sensors therefore must be able to detect the gradients from a phase shift of only $\sim 0.1$ radians representing the peak of the ice grain boundary against the background phase which would be approximately 40.6 radians in the center to greater than double that at the edge of the capsule a mm away.

For this article an analytical model for the grain boundary is assumed. The phase contribution due to the grain boundary, $\phi_{g b}$, is represented in analytical form by the expression $\phi_{g b}=\phi_{0}(\operatorname{abs}|\tanh (2 \pi x / 50)|-1)$, where $\phi_{0}$ is the peak amplitude of the phase from the grain boundary and $\mathrm{x}$ is the spatial coordinate in microns. The analytic phase gradient is expressed as $\left(8 \pi \phi_{0} / 50\right) /\left\{\exp (2 \pi x / 50)+\exp (-2 \pi x / 50)^{2}\right.$ for $\mathrm{x}$ greater than 0 . Based on these analytic expressions, the refraction angle, $\phi_{\text {refr }}$, of $\mathrm{x}$ rays passing through the ice grain boundary can be approximated as $\phi_{\text {refr }}=$ $\operatorname{atan}\left\{\left(4 \lambda \phi_{0} / 50\right) /\{\exp (2 \pi x / 50)+\exp (-2 \pi x / 50)\}^{2}\right\}$ for $\mathrm{x}$ greater than 0 . Fig. 1a shows the curve representing the analytic ice grain boundary with a $5 \mu \mathrm{m}$ depth as the solid black line. The analytic phase gradient is shown as a solid gray line in Fig. $1 \mathrm{a}$ as well and Fig. $1 \mathrm{~b}$ shows the analytic refraction angle of $\mathrm{x}$ rays passing through the grain boundary. The maximum refraction angle is $\sim 280 \mathrm{nrad}$ in this case. 

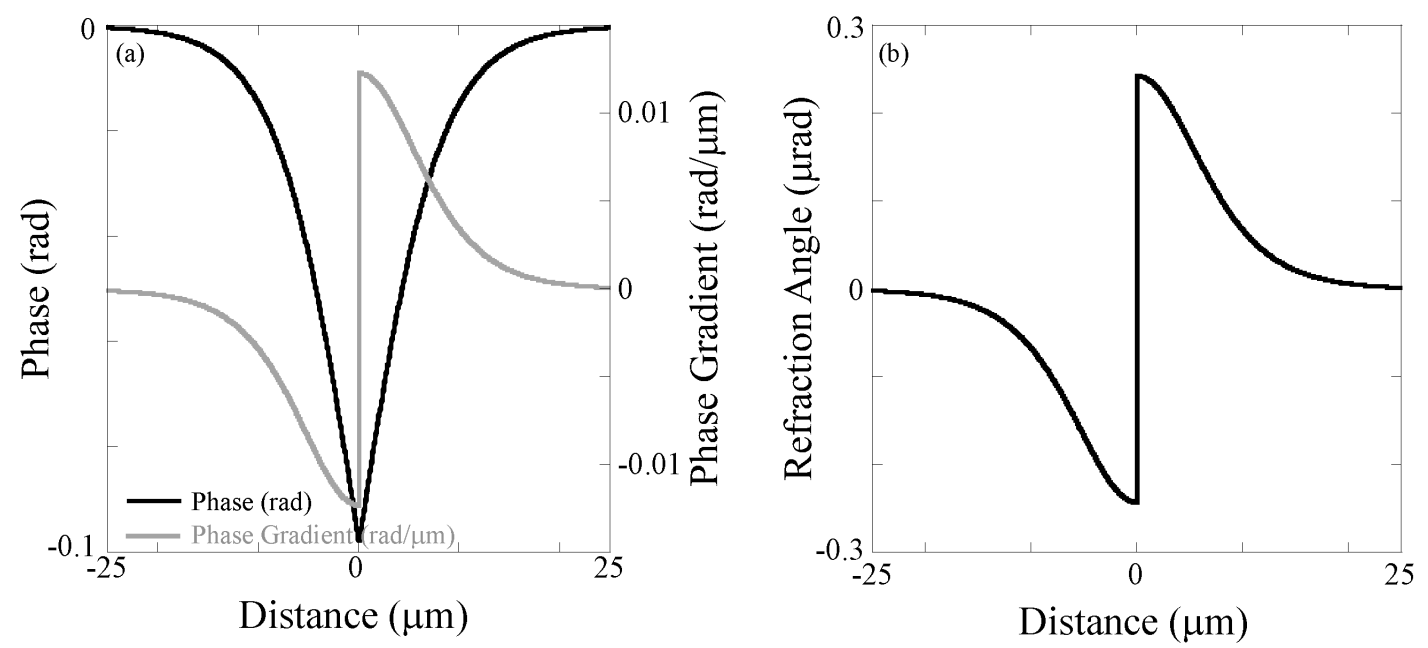

Fig. 1. Analytic model of the phase contribution due to the DT ice grain boundary. Fig. 1a represents the phase for an ice grain boundary with a $5 \mu \mathrm{m}$ depth as the solid black line and the corresponding phase gradient as the solid gray line. Fig. $1 \mathrm{~b}$ represents the corresponding refraction angle of $\mathrm{x}$-rays passing through the DT ice grain boundary.

\subsection{Simulated Experimental Setup}

The experimental measurement setup for the characterization of the fusion capsules consists primarily of a micro-focus $\mathrm{x}$-ray source, the fusion capsule itself, a phase flattener, the wavefront sensor, a filter and the detector as shown in Fig. 2. The micro-focus X-ray source will be assumed to contain a source size of approximately $5 \mu \mathrm{m}$ in diameter. Current experimental work with phase contrast imaging uses a micro-focus $\mathrm{x}$-ray source which has a source size of $5 \mu \mathrm{m}$ in diameter and a tungsten anode operating at $50 \mathrm{kV} .{ }^{5}$ The $\mathrm{L}$ shell emission is in the $8-11 \mathrm{keV}$ x-ray range. Operating the source at $50 \mathrm{kV}$ will result in significant bremsstrahlung radiation at higher x-ray energies. ${ }^{6,2}$ The detector, however, is an x-ray CCD camera which becomes optically thin to the higher energy photons. ${ }^{5}$ The lower energy x-rays can be removed and a narrower energy range within the L-shell emission can be selected by filtering with a thin foil such as copper.

A phase flattener is proposed to reduce the low order phase response from the capsule geometry. The phase of the fusion capsule itself will vary from approximately 45 radians in the center to more than double that at the edge of the capsule a mm away. This is not critical as the measurement will primarily concentrate on the central region of the fusion capsule with two additional orthogonal views to provide information on grain boundaries over the entire capsule. With this phase flattener in, however, the difference between the reference spot locations measured before the fusion capsule and flattener are inserted and the spot locations measured after the fusion capsule and flattener are inserted will provide a direct measurement of the rms surface roughness and the depth of the grain boundaries, providing a simple quantitative measure of the depth of the ice grain boundaries without even requiring a phase reconstruction as shown in section 3 . The phase flattener would then represent an inverse phase to the fusion capsule and could be placed immediately before or after the fusion capsule. 


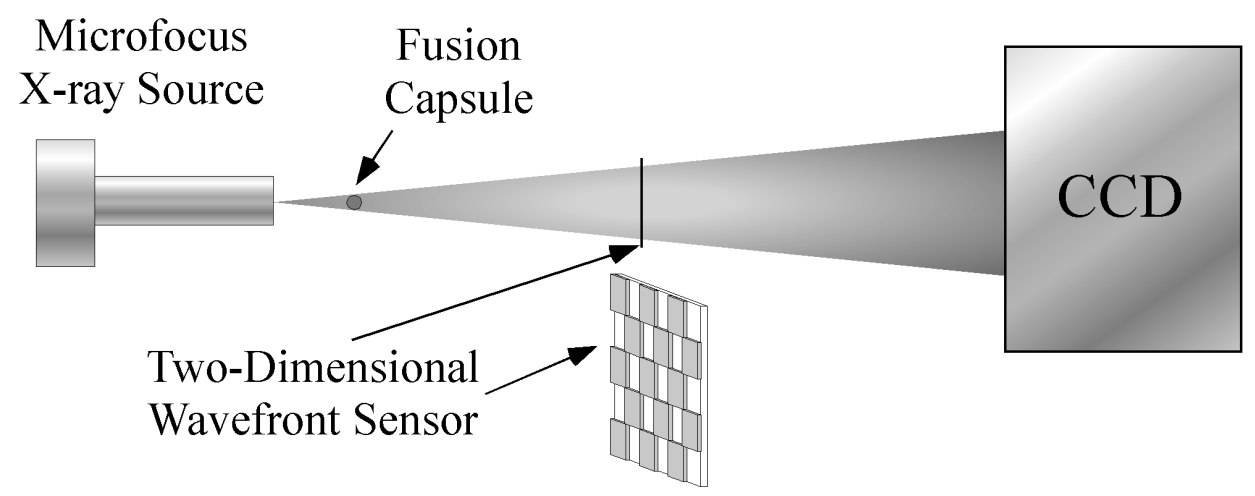

\section{Shearing \\ Interferometer}

Fig. 2. Experimental geometry, with a two-dimensional x-ray shearing interferometer, which would be used to measure the fusion capsule phase.

\subsection{Grating Design at $10 \mathrm{keV}$ for the Two-Dimensional X-Ray Shearing Interferometer}

The shearing interferometer uses orthogonal phase gratings which can be designed as either two crossed phase gratings or a single checkerboard pattern as it's two-dimensional wavefront sensor. The phase gratings are designed such that the even orders of the grating are eliminated. In order for the efficiency of the even orders, greater than the $\mathrm{m}=0$ order, of a transmission grating to go to zero at X-ray wavelengths, the width of the slits must be half of the grating pitch. ${ }^{7,8}$ In addition for the efficiency of the $\mathrm{m}=0$ order of the grating to go to zero, there must be negligible absorption and the bar structure of the grating must produce a shift of $\pi$ radians relative to the slits of the grating. ${ }^{7,8}$ Light elements can easily be made to provide the desired $\pi$ phase shift and yet not provide any significant absorption of the $\mathrm{x}$ rays. As a specific example we take Silicon and go through the design for a $10 \mathrm{keV}$ phase grating. For this example the width of the bar structure in the grating is identical to that of the slits such that all even orders above $\mathrm{m}=0$ go to zero. The Silicon bars are made with a thickness of $12.7 \mu \mathrm{m}$ such that a $\pi$ phase shift is achieved for $10 \mathrm{keV}$ x rays and the efficiency of the $\mathrm{m}=0$ order approaches zero. In the case of silicon there is some level of absorption which limits how much of the $\mathrm{m}=0$ order can be removed. The order efficiency as a function of x-ray energy for this grating is then shown in Fig. 3. At the design energy of $10 \mathrm{keV}$, the efficiency of the $\mathrm{m}=0$ order approaches zero and the efficiency of the $\mathrm{m}= \pm 1$ order is approximately $40 \%$. The efficiency of the odd orders goes approximately as $1 / \mathrm{m}^{2}$ with the $\mathrm{m}= \pm 3$ and \pm 5 shown in Fig. $3{ }^{7,8}$ The gratings can be manufactured in a similar manner to x-ray Talbot gratings. ${ }^{9}$ These particular gratings have a relaxed aspect ratio relative to the $\mathrm{x}$-ray Talbot gratings, $1.5 \mathrm{vs},>5$, and should therefore be simpler and cheaper to manufacture.

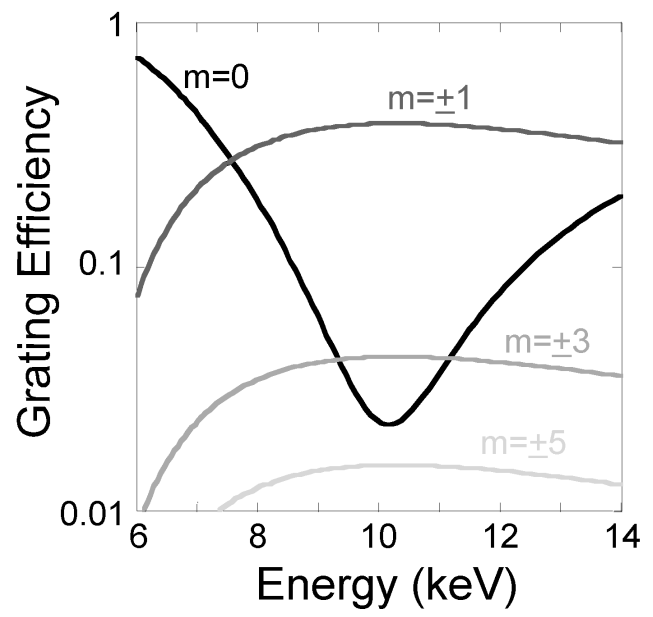


Fig. 3. Spectral order efficiency for a transmission grating composed of $12.7 \mu \mathrm{m}$ thick Silicon bars. This thickness provides the required $\pi$ phase shift at $10 \mathrm{keV}$ such that the zero order of the grating is minimized.

\section{SIMULATED PERFORMANCE OF THE WAVEFRONT SENSOR}

The simulations performed for this article are done so in collimated space in an analogous fashion to previous curvature wavefront sensor simulations. ${ }^{10,11}$ The simulation geometry is shown explicitly in Fig.4. The far right-hand side shows the geometry of the experiment in which a micro-focus $x$-ray source would reside in the location of the focus of the lens and illuminate the fusion capsule and $\mathrm{x}$-ray mask with a spherically diverging beam which would then be collected with the x-ray CCD camera. Each of these devices, the x-ray source, the fusion capsule, the x-ray mask and the x-ray CCD camera, has an object plane in collimated space on the left-hand side of the lens as shown in Fig. 4. Thus the simulation can be performed in collimated space with the appropriate magnification placed on each of the objects.

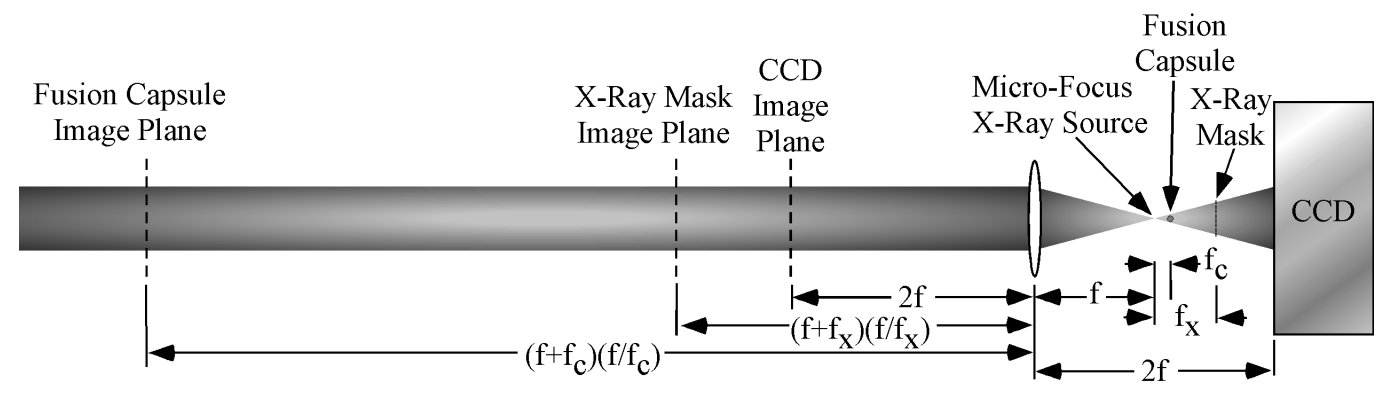

Fig. 4. Simulation geometry used to calculate the phase of the fusion capsule for the two-dimensional shearing interferometer performing Fresnel propagation in collimated space.

For the simulations, the following assumptions are made; 20 micron pixels on the $\mathrm{CCD}, 40$ micron pitch on the $\mathrm{x}$-ray gratings(in collimated space), $\mathrm{fc}=0.1 \mathrm{~m}, \mathrm{fx}=0.2 \mathrm{~m}$ and $\mathrm{f}=1.1 \mathrm{~m}$. In an actual experiment the gratings would have a pitch of 17.8 microns in the spherically expanding x-ray beam which would give them a modest aspect ratio, $\sim 1.5$, in comparison to $\mathrm{x}$-ray Talbot gratings. Given these assumptions, the fusion capsule is magnified a factor of 2 onto the $\mathrm{x}-$ ray mask and a factor of 10 onto the $\mathrm{x}$-ray CCD camera. The capsule is $2 \mathrm{~mm}$ in diameter, which would require a CCD with an active area of at least $2 \mathrm{~cm}$. This is consistent with 1024 pixels at 20 microns/pixel. The x-ray CCD will measure the wavefront gradient at a scale of 4 microns on the capsule ( 40 microns in collimated space). The simulations are performed on one quarter of the fusion capsule, $1 \mathrm{~mm} \times 1 \mathrm{~mm}$, such that across the simulation box there are 512 pixels on the $\mathrm{x}$-ray $\mathrm{CCD}$ with each pixel representing an area of $4 \mathrm{x} 4$ simulation pixels. Each grating feature, 40 microns, represents an area on the x-ray CCD of $2 \times 2$ CCD pixels or a total of 64 simulation pixels. The simulations are performed with both read noise and Poisson noise. For the simulations shown the read noise was assumed to be $20 \mathrm{e}-\mathrm{rms}$ and the $\mathrm{x}-$ rays were assumed to produce 100,000 photons per every four x-ray CCD pixels.

The simulations begin by defining a uniform field at the image plane of the fusion capsule located at the left-hand side of Fig.4 and assuming a point x-ray source. The simulations were performed with 2048 by 2048 simulation pixels covering a range on the camera of $\sim 5$ microns per simulation pixel, representing $\sim 0.5$ microns/sim.pix. on the fusion capsule itself. One quarter of the fusion capsule sphere was simulated with an initial phase profile of $0.026 \mathrm{rad}$ RMS, $\sim 0.5 \mu \mathrm{m}$ RMS, placed on the fusion capsule to simulate surface roughness. A Kolmogorov turbulence profile was assumed for the surface roughness of the DT ice layer. In addition to the phase profile representing the surface roughness of the DT ice, six DT ice grain boundaries were placed across the fusion capsule with each one having a width of 50 microns(500 microns in collimated space) but different lengths, depths and angles, as shown in Fig. 6a. In particular a long horizontal and vertical ice grain boundary were introduced which had a 5 micron depth. The phase representing the fusion capsule and phase flattener were then used to construct a new field which was then Fresnel propagated to the twodimensional x-ray transmission grating shown in Fig. 5a. The periodic phase pattern representing the crossed phase grating is then added to the field and the field is propagated to the x-ray CCD camera. When a periodic structure is 
placed in a beam, images of that structure will appear downstream of the object as discovered by Talbot. ${ }^{12}$ More precisely if a phase grating is placed in the beam composed of alternating equal width bars of 0 and $\pi$ phases, then the field at the location of the phase structure will be reproduced a distance $\mathrm{d}_{\mathrm{T}}=\mathrm{d}^{2} / 2 \lambda$ downstream of the phase structure. In this expression, $d_{T}$ is the Talbot distance, $d$ represents the pitch of the phase grating and $\lambda$ is the wavelength of the source. At a distance equal to $\mathrm{d}_{\mathrm{T}} / 4$ and $3 \mathrm{~d}_{\mathrm{T}} / 4$, the initial phase pattern across the beam has become uniform and the initially uniform intensity has acquired the periodic structure of the initial phase pattern with the pitch of the intensity pattern equal to half that of the original phase grating. At a distance of $\mathrm{d}_{\mathrm{T}} / 2$, the phase pattern is reversed from the original phase grating and the intensity pattern is uniform such that this particular location can not be used for wavefront sensing. The field at the x-ray CCD camera, assuming an initial point x-ray source, forms an array of spots on the camera. as shown in Fig. 5b. One method to simulate a finite source size is to convolve the spot pattern from the point source with the geometric projection of the source onto the x-ray CCD. The source is magnified by a factor of five onto the CCD relative to the position of the shearing grating. Fig. $5 \mathrm{c}$ shows the 25 micron circle that is convolved with the point source spot pattern. Fig. $5 \mathrm{~d}$ shows the resultant spots on the x-ray CCD after the convolution has been performed.
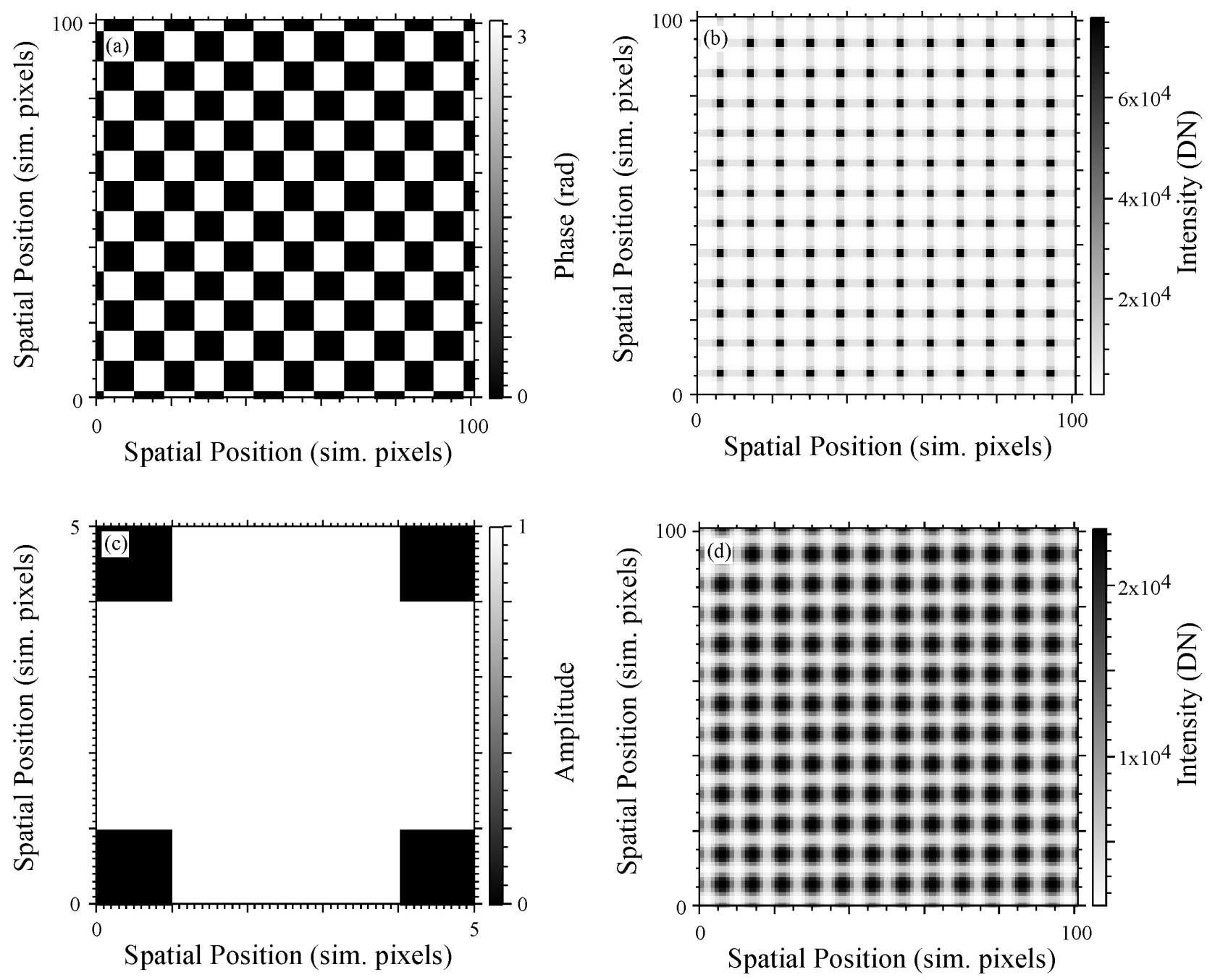

Fig. 5. Simulating the x-ray shearing interferometer. Fig. 5a represents the phase profile of the two-dimensional grating. As the $\mathrm{x}$-rays from a point source pass through the grating and travel to the CCD, they form spots on the CCD as shown in Fig. 5b. One method to simulate a finite source size is to convolve the spot pattern from the point source with the geometric projection of the source onto the $\mathrm{x}$-ray CCD. The source is magnified by a factor of five onto the CCD relative 
to the position of the shearing grating. Fig. $5 \mathrm{c}$ shows the 25 micron circle that is convolved with the point source spot pattern. Fig. $5 \mathrm{~d}$ shows the resultant spots on the x-ray CCD after the convolution has been performed.

The two-dimensional array of spots on the x-ray CCD measures the entire x-ray field. The amplitude of the spots is a measure of the amplitude of the electric field and the displacement of the spots is a measure of the phase of the x-ray electric field at the wave-front sensor. Performing the simulation with and without a fusion capsule/flattener, as would be done for an experimental measurement, allows one to do a simple difference of the two images to see a quantitative measure of the gradients as can be seen in Fig. 6b. A phase reconstruction can be performed by first finding the exact locations of the reference spots and the spots due to the introduction of the fusion capsule/flattener into the system. For these simulations the grating was chosen such that 4 CCD pixels were used to measure each spot and as such a simple center-of-mass centroiding algorithm was used to determine the spot locations. If more CCD pixels are used for each spot them more advanced centroiding algorithms could be employed. ${ }^{13}$ Once the locations of each of the spots is determined, then the reference spots are subtracted from the spots due to the introduction of the fusion capsule/flattener into the system to determine the two-dimensional wavefront gradient. Given the two-dimensional wave-front gradients, the two-dimensional phase was reconstructed using a least squares multigrid wavefront reconstructor. ${ }^{14}$ The reconstructed wavefront, along with the measured x-ray field amplitude is then back-propagated to the fusion capsule image plane. The resultant phase is then shown in Fig. 7a, which compares favorably with the original phase shown in Fig. 6a. Specifically, Fig. 7b shows lineouts across two of the reconstructed DT ice grain boundaries, averaged along the length of the grain boundary. The dashed black line represents the analytic phase profile applied to the vertical and horizontal ice grain boundaries in Fig. 6a, as labeled in Fig. 7a. The light gray line represents the reconstruction of the long horizontal DT ice grain boundary seen in Fig. 7 a and the dark gray line represents the reconstruction of the vertical DT ice grain boundary seen in Fig. 7 a.
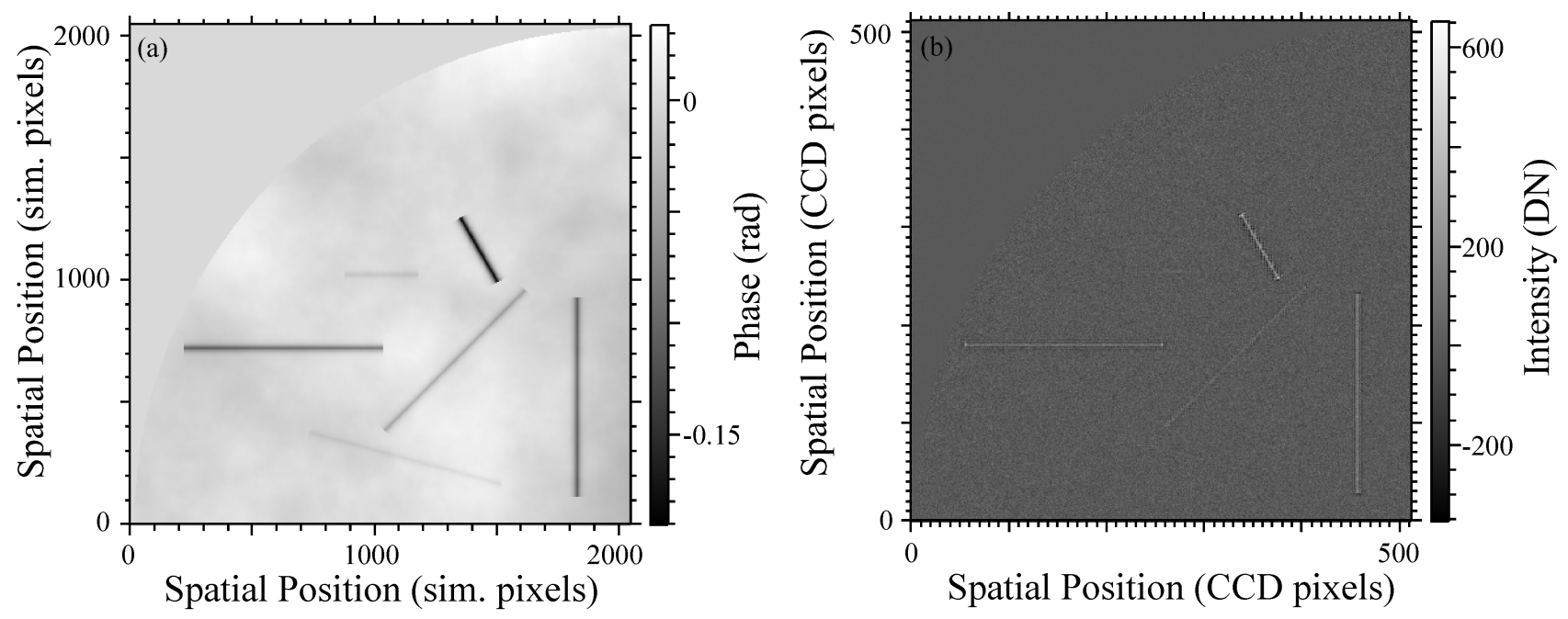

Fig. 6. Phase profiles and CCD difference images resulting from the simulations. Fig. 6a represents the input phase which includes six simulated grain boundaries on top of an 0.0098 rad RMS phase aberration representing the DT ice surface roughness. Fig. $6 \mathrm{~b}$ shows the difference between the images on the x-ray CCD with no fusion capsule in the metrology setup and with a fusion capsule and phase flattener in the shearing interferometer. 

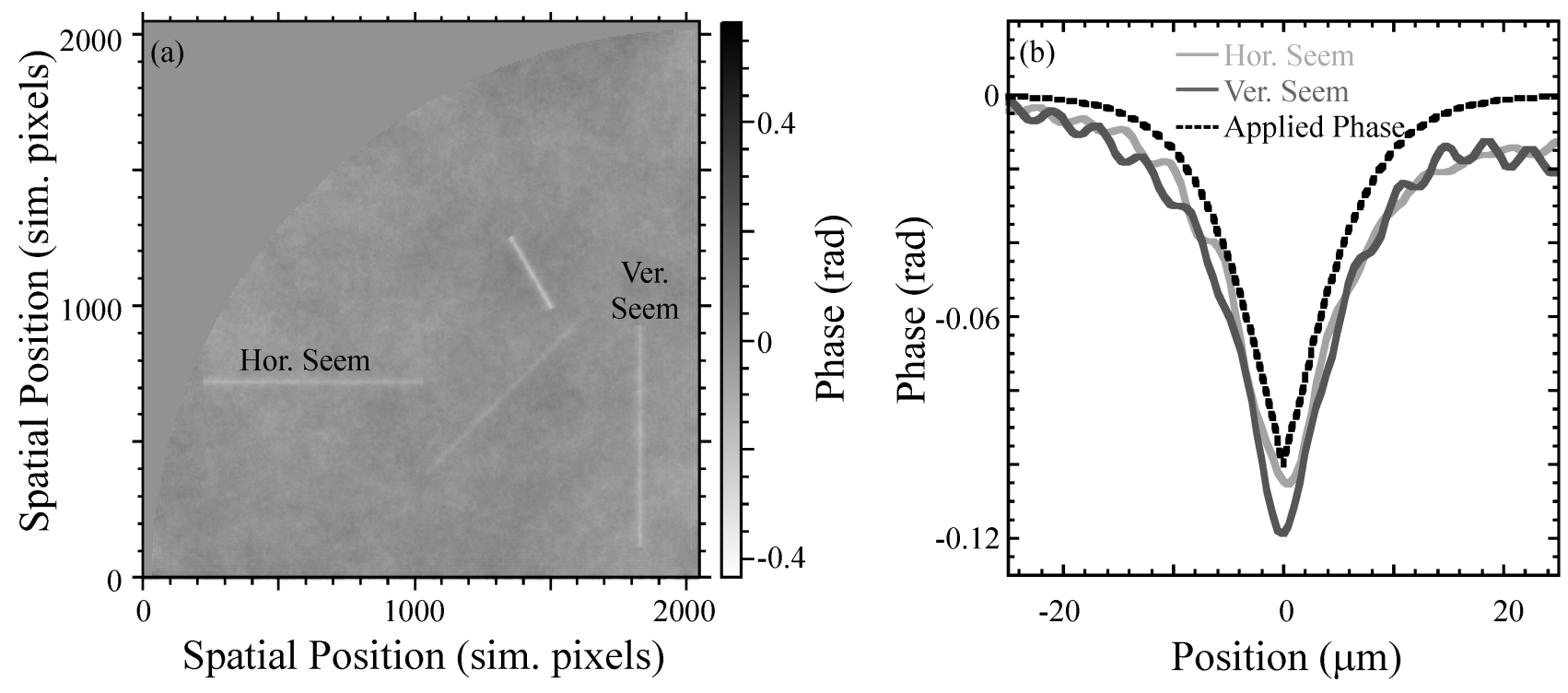

Fig. 7. Reconstructed phase profiles from the simulation. Fig. 7a shows the reconstructed phase resulting from the simulation with noise included in the simulations. Fig. 7b shows lineouts across two of the reconstructed DT ice grain boundaries, averaged along the length of the grain boundary. The dashed black line represents the analytic phase profile applied to the vertical and horizontal grain boundaries in Fig. 6a. The light gray line represents the reconstruction of the long horizontal DT ice grain boundary seen in Fig. 7 a and the dark gray line represents the reconstruction of the vertical DT ice grain boundary seen in Fig. 7 a.

\section{SUMMARY}

The performance of a two-dimensional x-ray shearing interferometer was simulated in the context of x-ray metrology on a fusion capsule using a finite source micro-focus $\mathrm{x}$-ray tube. This application takes advantage of the large disparity between the absorption coefficient and the phase shift component of light elements to measure the phase profile of a fusion capsule. The fusion capsule was simulated with six DT ice grain boundaries with the shortest DT ice grain boundary height of $0.015 \mathrm{rad}$, corresponding to a DT ice layer height of 0.75 microns, and the tallest DT ice grain boundary height of $0.196 \mathrm{rad}$, corresponding to a DT ice layer height of 10 microns. Two DT ice grain boundaries were simulated with a height of 5 microns or 0.098 radians and the reconstructed wave-fronts of these were compared directly with the analytic ice grain boundary initially imposed on the simulation. The peak height agreed within $\sim 10 \%$ and the full-width-at-half-maximum agreed to within $\sim 14 \%$ between the analytic phase profile and the two reconstructed phase profiles. This indicates that the two-dimensional shearing interferometer could be used in this application to measure DT ice grain boundaries and determine if their height exceeded the maximum grain boundary depth that can be tolerated on a fusion shot, $\sim 5 \mu \mathrm{m}$, without unacceptably impacting the yield.

\section{ACKNOWLEDGEMENTS}

The authors would like to acknowledge useful discussions with Alex Hamza, John S. Taylor and Bernard Kozioziemski. This work was performed under the auspices of the U.S. Department of Energy by Lawrence Livermore National Laboratory under Contract DEAC52-07NA27344.

\section{REFERENCES}


[1] A. Hamza, Private communication on fusion capsule performance (2010).

[2] B. J. Kozioziemski, J. D. Sater, J. D. Moody, J. J. Sanchez, R. A. London, A. Barty, and Jr. H. E. Martz, “X-ray imaging of cryogenic deuterium-tritium layers in a beryllium shell," Journal of Applied Physics 98, 103105 (2005).

[3] K.L. Baker, "X-Ray wavefront analysis and phase reconstruction with a two-dimensional shearing interferometer," Opt. Eng. 48 (8), 086501 (2009).

[4] K.L. Baker, Patent No. 12/794,312 (June 4, 2010).

[5] Bernie Kozioziemski, Private communication on fusion capsule and microfocus X-ray source parameters (2010).

[6] M. H. Uunsworth and J. R. Greening, "Theoretical Continuous and L-Characteristic X-Ray Spectra for Tungsten Target Tubes Operated at 10 t o 50 k,” PHYS. MED. BIOL. 15, 621 (1970).

[7] H. W. Schnopper, L. P. Van Speybroeck, J. P. Delvaille, A. Epstein, E. KIllne, R. Z. Bachrach, J. Dijkstra, and L. Lantward, "Diffraction grating transmission efficiencies for XUV and soft x rays," Applied Optics 16 (4), 1088 (1977).

[8] Michael C. Hettrick, Michael E. Cuneo, John L. Porter, Larry E. Ruggles, Walter W. Simpson, Mark F. Vargas, and David F. Wenger, "Profiled bar transmission gratings: soft-X-ray calibration of new Kirchoff solutions," Applied Optics 43 (19), 3772 (2004).

[9] Masatake Matsumoto, Kinji Takiguchi, Makoto Tanaka, Yoichi Hunabiki, Hiroaki Takeda, Atsushi Momose, Yuichi Utsumi, and Tadashi Hattori, "Fabrication of diffraction grating for X-ray Talbot interferometer," Microsyst. Technol. 13, 543-546 (2007).

[10] Francois Roddier and Claude Roddier, "Wave-front reconstruction from defocused images and the testing of ground-based optical telescopes," J. Opt. Soc. Am. A. 10 (11), 2277 (1993).

[11] Anastacia M. Manuel, Donald W. Phillion, Scot S. Olivier, Kevin L. Baker, and Brice Cannon, "Curvature wavefront sensing performance evaluation for active correction of the Large Synoptic Survey Telescope (LSST)," Optics Express 18 (2), 1528 (2010).

[12] F. Talbot, "Facts relating to optical science IV," Philos. Mag. 9, 401 (1836).

[13] K.L. Baker and M.M Moallem, "Iteratively Weighted Centroiding for Shack-Hartmann Wave-front Sensors Optics," Express 15, 5147 (2007).

[14] K. L. Baker, "Least-Squares Wave-Front Reconstruction of Shack-Hartmann Sensors and Shearing Interferometers using Multigrid Techniques,” Rev. Scien. Instr. 76, 053502 (2005). 